\title{
CALIBRATION OF LOW COST RGB AND NIR UAV CAMERAS
}

\author{
A. Fryskowska ${ }^{\text {a }}$, M. Kedzierski ${ }^{\text {a }}$, A. Grochala ${ }^{\text {a }}$, A. Braula ${ }^{\text {a }}$ \\ ${ }^{a}$ Department of Remote Sensing and Photogrammetry, Faculty of Civil Engineering and Geodesy, \\ Military University of Technology, 2 Kaliskiego str. Warsaw, Poland \\ (anna.fryskowska, michal.kedzierski, aleksandra.grochala@wat.edu.pl; bralla@o2.pl)
}

\section{Commission I, ICWG I/Vb}

KEY WORDS: calibration, non-metric camera, calibration tests, GRD, GSD, low-cost cameras

\begin{abstract}
:
Non-metric digital cameras are being widely used for photogrammetric studies. The increase in resolution and quality of images obtained by non-metric cameras, allows to use it in low-cost UAV and terrestrial photogrammetry. Imagery acquired with non-metric cameras can be used in 3D modeling of objects or landscapes, reconstructing of historical sites, generating digital elevation models (DTM), orthophotos, or in the assessment of accidents.

Non-metric digital camcorders are characterized by instability and ignorance of the interior orientation parameters. Therefore, the use of these devices requires prior calibration. Calibration research was conducted using non-metric camera, different calibration tests and various software.

The first part of the paper contains a brief theoretical introduction including the basic definitions, like the construction of non-metric cameras or description of different optical distortions. The second part of the paper contains cameras calibration process, details of the calibration methods and models that have been used. Sony Nex 5 camera calibration has been done using software: Image Master Calib, Matlab - Camera Calibrator application and Agisoft Lens. For the study 2D test fields has been used. As a part of the research a comparative analysis of the results have been done.
\end{abstract}

\section{INTRODUCTION}

Currently, the most common and most frequently used in the Unnmaned Aerial Systems (UAS) are compact digital cameras (non-metric cameras). Metric cameras are able to guarantee the stability of the internal orientation elements. Typically, these cameras do not have the possibility of zoom lens. In contrast, non-metric cameras are characterized by instability and ignorance of the elements of internal orientation.

Cameras used for photogrammetric purposes are usually compact cameras or SLR (Single-Lens Reflex Camera).

Compact cameras are cameras with a small size and simple design, equipped with a fixed lens (fixed focus lens or zoom lens). The lens retracts into the camera body. Focus or exposure time is carried out automatically. The viewfinder is placed above the lens, causing the phenomenon of parallax. These cameras have a much smaller matrices than an SLR or hybrids . compact Cameras having a fixed lens have smaller geometric resolution than cameras having a zoom lens .

While the design of SLR cameras now prevails among the top and middle class. There is only one lens, which is used at the same time to observe the frame and exposures. The advantage of this type of camera is to give the frame in the form in which they will be exposed. Another advantage is the total lack of parallax. Lenses with large focal lengths have built-in stabilizers, which allow you to make sharp images without a tripod. The SLR lenses are used so-called zooms and fixed lens.

Digital camera consists of elements such as: body, bayonet lens, image sensor, LCD display, memory card.

The matrix is the arrangement of light sensitive elements. The task of each element is registration and ability to read the electrical signal that is proportional to the amount of incident light to the matrices. The digital cameras are most frequently used color filters so that each of the sensor reads the intensity of the predetermined width of the light spectrum.

Matrices are divided into two types of structures of their construction: CCD (ang. Charge Coupled Device) and CMOS (ang. Complementary Metal-Oxide-Semiconductor). They differ in production technology. The CCD has one converter (analogdigital) that processes information from each pixel into a digital signal. Data is read pixel by pixel. However, in the CMOS sensor, each pixel has its own transmitter, so that the matrix is faster (faster recording images and the ability to perform more shots). The array has on its surface a greater amount of circuitry that can block light entering the inside of the pixel.

More and more the amateur digital cameras are used for photogrammetric purposes. Camera selection (metric or nonmetric) depends on the desired accuracy.

For topographical applications, architectural inventory, or in cases where it is required to obtain measurements with very high accuracy, photogrammetric cameras are used (metric). In contrast, digital compact cameras are used in cases where they may get lower measurement accuracy for example when measured special objects. The use of digital cameras for photogrammetric purposes requires calibration. This is due to the fact that the non-metric cameras elements of internal orientation are unknown. In connection with the increase in resolution and quality of images obtained with non-metric cameras they are used in aerial and terrestrial photogrammetry. Non-metric images can be used for modeling 3D objects, reconstruction of monuments, to generate digital elevation models (DEM), orthophotos, or for the assessment of accidents.

\footnotetext{
* Corresponding author
} 


\section{RELATED WORKS}

Camera calibration and the evaluation of high-quality interior orientation parameters is one main topic in research and development of photogrammetry since decades. (Hastedt and Luhmann, 2015). Architectural documentation is carried out mostly with non-metric cameras or video-cameras. Such experiences have been shown in (Delis et al., 2013). Unknown internal geometry is a main problem in this context, particularly for wide-angle lenses with their considerable amount of distortion. Some aspects of calibration of non-metric cameras were presented in (Karras and Mavrommati, 2001). Other publications that threats about the calibration of non-metric camera calibration methods are (Fraser and Remondino, 2006), (Honkavaara et al., 2006), (Harley et al., 2006), (Kedzierski and Fryskowska, 2008) or (Wang et al., 2009). The work that presents results of surveying two objects of interest for the conservation of the architectural and cultural patrimony of the Old Havana with the final verification of results is presented in (Rodríguez et al., 2008).

Currently, non-metric cameras are mounted in the Unmanned Aerial Systems. In this case, also interior orientation information is needed. Such experiences describe (Pérez et al. 2011), (Douterloigne et al., 2009), (Kedzierski, M., Wierzbicki, 2015), (Dabrowski et al., 2014)

\section{OPTICAL DISTORTIONS}

Defects (optical errors) camera lenses include aberrations: chromatic aberration, spherical, coma, field curvature, distortion and astigmatism.

\subsection{Lens distortion}

In non-metric cameras, there are two types of distortion: radial and tangential (tangent). The device, which is ideal lens angle at which the beam enters main radius (it is one that after passing through the lens is parallel to the incident beam) is equal to the angle at which the beam exits.

The distortion is related to the structure of the lens - the distribution and the value is already known in the design stage of the lens, so the radial distortion is defined as the theoretical distortion. The value of distortion $\Delta \mathrm{r}$ depends on field angle (the angle between the axis of the lens and the main radius) and the radial distance $r$.

$$
\Delta r=r-f \tan \propto
$$

where: $\Delta \mathrm{r}-$ radial distortion;

$r$ - radial distance;

$\mathrm{f}$ - focal length;

$\alpha$ - field angle.

The curve of the radial distortion can be represented using a polynomial of odd powers of the radial distance.

$$
\Delta r=K_{1} r^{3}+K_{2} r^{5}+K_{3} r^{7}+\cdots
$$

where: $\mathrm{K}_{1}, \mathrm{~K}_{2}, \mathrm{~K}_{3}$ - polynomial coefficients of distortion; $\mathrm{r}$ - radial distance.

$$
r=\sqrt{\left(x-x_{0}\right)^{2}+\left(y-y_{0}\right)^{2}}
$$

where: $\quad x, y$ - image coordinates of the point;

$\mathrm{x}_{0}, \mathrm{y}_{0}$ - image coordinates of the principal point.
To describe the radial distortion curve we need to know only two components of polynomial distortion. For analytical description it is required to determine a radial distortion coefficients: K1, K2 and K3.

$$
\begin{aligned}
& \Delta x_{r}=\bar{x}\left(K_{1} r^{2}+K_{2} r^{4}+K_{3} r^{6}+\cdots\right) \\
& \Delta y_{r}=\bar{y}\left(K_{1} r^{2}+K_{2} r^{4}+K_{3} r^{6}+\cdots\right)
\end{aligned}
$$

where: $\Delta \mathrm{x}_{\mathrm{r}}, \Delta \mathrm{y}_{\mathrm{r}}$ - the effect of radial distortion for image coordinates $\mathrm{x}, \mathrm{y}$;

$\mathrm{y}^{-}, \mathrm{x}^{-}-$image coordinates relative to the principal point.

Radial distortion in the real lens is not strictly symmetrical (different from the theoretical distortion). For different radii of radial distortion do not coincide with each other.

\section{METHODS OF NON-METRIC CAMERAS CALIBRATION}

For non-metric cameras the elements of internal orientation are instable and unknown. Any type of conventional camera, which does not require any additional parameters (fiducial marks) to define the internal orientation are referred to as non-metric cameras.

The main problem of non-metric images is that they do not guarantee us stability elements of the interior orientation (their repeatability). Measurement of fiducial marks associated with the principal point is impossible, because they do not occur in images taken by the non-metric digital camera. The role of fiducial marks in the images of the non-metric cameras perform extreme corners of the image pixels.

The process of determination of internal orientation parameters is calibration. During calibration we determine: focal length, principal point, radial distortion, tangential distortion, and other. (Harley et al., 2005)

There are several methods of camera calibration: laboratory, field, self-calibration.

Laboratory calibration requires the calibrator and usually is applied for the metric cameras. The file calibration method requires known reference filed. The method bases on the images of this test field. The test field consists of a large number of points that evenly cover the entire frame. Signalized points are imaged in the picture. There are flat test fields - 2D and spatial 3D. Self-calibration is the process of camera calibration performed during the measurement process. This is due to the increased number of images taken, and the necessity of the use of specialized software. The greater the number of images, and thus the greater the number of measured homologous points, the accuracy is higher.

\subsection{Cameras used}

Two non-metric cameras were used: SONY NEX 5 with two types of matrices: RGB (SONY NEX 5R) and NIR (SONY NEX 5T). Both cameras have the same structure like Sony NEX5 compact camera, with the APS-C Exmor CMOS matrix (format - 3:2) with the maximum resolution of $4912 \times 3264$. The size of the matrix is 23,5 x 15,6 mm (Figure 1). 


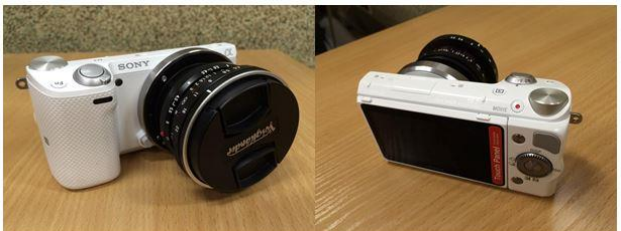

Figure 1. Sony NEX 5T camera with NIR matrix

The camera has the ability to exchange the lens. In the camera lens Voigtlander $15 \mathrm{~mm} \mathrm{f} \mathrm{/} 4.5$ is mounted. It is a wide-angle lens. It has a field of view equal to $110^{\circ}$. It does not have stability.

Cameras Sony NEX 5R and Sony NEX 5T differ from each with the matrix structure. In both cases, it has been used CMOS APS - C matrix. The matrix is composed of millions of detectors - light-sensitive elements. Each of the pixels are equally sensitive to the wavelength of electromagnetic data. That the camera can capture an image in color on a matrix Bayer filter has been applied. The camera Sony NEX 5R has a Bayer filter (Figure 2), which transmits light at three different wavelengths (RGB).

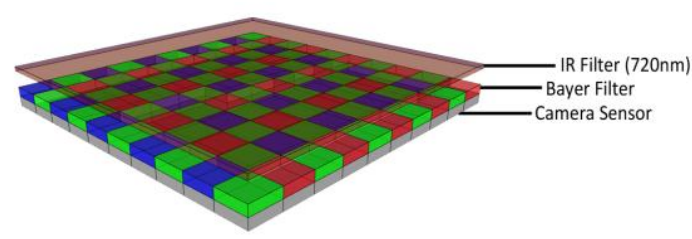

Figure 1. Bayer filter (Nikon, 2016)

Two green pixels falls one pixel red and blue. To create a multicoloured image of this combination of points, interpolation Bayer have to be used. It bases on the estimation of the light intensity measured by the neighbouring pixels, missing information for a particular colour. The camera therefore operates in the visible range from 400 to $720 \mathrm{~nm}$.

However, in the case of cameras Sony NEX 5T cell sensitive to the colour blue is replaced with a cell sensitive to NIR range, so the camera takes the image in the range from 500 to $1200 \mathrm{~nm}$. In addition, in the camera additional filter for transmitting infrared is mounted. In this case, the selected filter is HOYA R72 with a diameter of $55 \mathrm{~mm}$ (Figure 3). It is a filter which absorbs all visible light to a length of $720 \mathrm{~nm}$, and transmits infrared radiation.

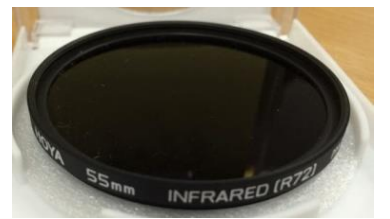

Figure 2. infrared filter $720 \mathrm{~nm}$

\section{RESEARCH}

There were used three different calibration algorithms implemented in different software: Image Maser Calib, Matlab and Agisoft.

5.1. Image Master Calib enables determination of 7 calibration parameters: focal length $f$, principal point coordinates $\mathrm{x}_{0}, \mathrm{y}_{0}$, radial distortion coefficients: $\mathrm{K}_{1}, \mathrm{~K}_{2}$ and tangential distortion coefficients: $\mathrm{P}_{1}, \mathrm{P}_{2}$. The test filed consist of 5 squares and 145 black points (Figure 4):

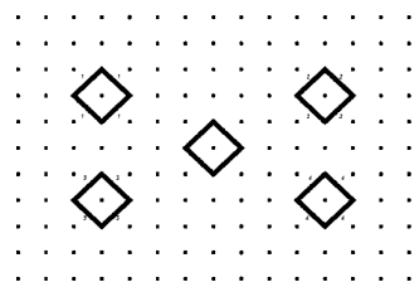

Figure 4. Test filed for Image Master Calib

Calibration is conducted using a semi-automatic or automatic method. The condition of proper calibration are values of the standard deviation of image coordinates of all measured points. This values should not exceed the value of one pixel.

The calibration report contains information of the calibration parameters.

In addition the pixel size [microns], and the program displays a graphical interpretation of the distortion of the image.

5.2. Matlab R2015a - Camera Calibrator software was created in order to determine the parameters of the interior orientation of digital cameras (non-metric). It enables to determine the eight calibration parameters: focal length $f_{x}, f_{y}$, principal point coordinates $\mathrm{x}_{0}, \mathrm{y}_{0}$, radial distortion coefficients: $\mathrm{K}_{1}, \mathrm{~K}_{2}$ and tangential distortion coefficients: $\mathrm{P}_{1}, \mathrm{P}_{2}$.

The test field reminds chessboard (Figure 5). The test consists of a squares: white and black, which are arranged alternately. One page should contain an even number of fields, and the other odd. In this way, the pattern comprises two black corners along one side and white two corners on the opposite side. The calibration sheet cannot take the shape of a square, as arranged along a long side axis $\mathrm{X}$, but along the short axis $\mathrm{Y}$. This allows to specify the orientation of the pattern.

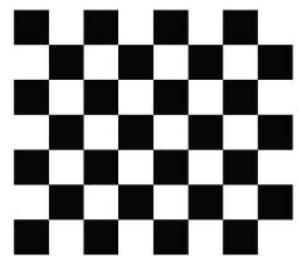

Figure 5. Test field for Camera Calibrator

Images of the test field can be added directly from the camera (set camera parameters to check the pictures) or in the folder. It is required to set the dimension of the side of the square chessboard. The program analyzes images to determine whether they meet the requirements of the calibrator and then detects points.

5.3. Agisoft Lens consist of the modules: Agisoft PhotoScan, Agisoft Lens and Agisoft StereoScan. For the purpose of calibration (flat test field) is used for application Agisoft Lens. However Agisoft software also has the ability to perform selfcalibration for non-metric images in two other modules (Agisoft PhotoScan and Stereoscan). In this software we can determine the same calibration parameters like in the Camera Calibrator application. As in the case of the Camera Calibrator for calibration chessboard is used. 


\section{RESULTS}

10 series of images have been taken with Sony NEX cameras, which resulted in 100 images per test field. The calibration images should be taken under similar conditions to the field samples for most accurate results. (Pérez et al., 2011)

Calibration have been done in three mentioned software.

The results of two non-metric cameras calibrations in Image Master (interior orientation parameters) are presented in Tables 1 and 2 .

\begin{tabular}{|l|c|c|}
\hline Parameter & Mean value & Standard deviation \\
\hline $\mathbf{f}[\mathbf{m m}]$ & 14.957 & 0.002 \\
$\mathbf{x}_{\mathbf{0}}[\mathbf{m m}]$ & 11.239 & 0.005 \\
$\mathbf{y}_{\mathbf{0}}[\mathbf{m m}]$ & 7.343 & 0.022 \\
$\mathbf{K}_{\mathbf{1}}$ & $2.11 \mathrm{E}-04$ & $1.82 \mathrm{E}-06$ \\
$\mathbf{K}_{\mathbf{2}}$ & $-5.38 \mathrm{E}-07$ & $6.56 \mathrm{E}-09$ \\
$\mathbf{P}_{\mathbf{1}}$ & $-2.34 \mathrm{E}-05$ & $3.15 \mathrm{E}-06$ \\
$\mathbf{P}_{\mathbf{2}}$ & $7.32 \mathrm{E}-05$ & $3.36 \mathrm{E}-06$ \\
\hline
\end{tabular}

Table 1. The results of Sony NEX 5R calibration (Image Master)

\begin{tabular}{|l|c|c|}
\hline Parameter & Mean value & Standard deviation \\
\hline $\mathbf{f}[\mathbf{m m}]$ & 14.962 & 0.002 \\
$\mathbf{x}_{\mathbf{0}}[\mathbf{m m}]$ & 11.352 & 0.007 \\
$\mathbf{y}_{\mathbf{0}}[\mathbf{m m}]$ & 7.512 & 0.014 \\
$\mathbf{K}_{\mathbf{1}}$ & $2.09 \mathrm{E}-04$ & $1.29 \mathrm{E}-06$ \\
$\mathbf{K}_{\mathbf{2}}$ & $-5.64 \mathrm{E}-07$ & $2.62 \mathrm{E}-10$ \\
$\mathbf{P}_{\mathbf{1}}$ & $-4.82 \mathrm{E}-05$ & $4.51 \mathrm{E}-06$ \\
$\mathbf{P}_{\mathbf{2}}$ & $-6.05 \mathrm{E}-05$ & $3.36 \mathrm{E}-06$ \\
\hline
\end{tabular}

Table 2. The results of Sony NEX 5T calibration (Image Master)

Constant $f$ for both cameras has been determined with very high accuracy, as presented by the low standard deviation values. The calculated value is different from the theoretical manufacturer's focal length of $15 \mathrm{~mm}$ by $0.04 \mathrm{~mm}$. In the case of the other theoretical parameters manufacturer did not give their value, and therefore they can not be compared.

When comparing the values obtained for the two non-metric cameras the same manufacturer can be stated that the results do not differ much from each other. Focal length of both cameras have reached an almost identical value that is $14.96 \mathrm{~mm}$. In the case of principal point coordinates two main values differ from each oher by the tenth millimetre. It should be noted that the standard deviation of these values are small - amount to hundredths of millimetre. Also very low standard deviations for the second factor of a polynomial has been reached.

In comparison, in Tables 3 and 4 calibration results for two cameras in two other software packages (Matlab and Agisoft) have been presented.

\begin{tabular}{|l|c|c|}
\hline \multicolumn{3}{|c|}{ Matlab } \\
\hline \multicolumn{1}{|c|}{ Parameter } & Mean value & Standard deviation \\
\hline $\mathbf{f}[\mathbf{m m}]$ & 14.985 & 0.083 \\
$\mathbf{x}_{\mathbf{0}}[\mathbf{m m}]$ & 11.421 & 0.120 \\
$\mathbf{y}_{\mathbf{0}}$ [mm] & 7.518 & 0.125 \\
$\mathbf{K}_{\mathbf{1}}$ & -0.001 & $1.43 \mathrm{E}-04$ \\
$\mathbf{K}_{\mathbf{2}}$ & 0.003 & $1.47 \mathrm{E}-03$ \\
$\mathbf{K}_{\mathbf{3}}$ & -0.011 & $4.61 \mathrm{E}-03$ \\
$\mathbf{P}_{\mathbf{1}}$ & $3.35 \mathrm{E}-06$ & $1.17 \mathrm{E}-06$ \\
$\mathbf{P}_{\mathbf{2}}$ & $2.52 \mathrm{E}-06$ & $1.4 \mathrm{E}-06$ \\
\hline \multicolumn{3}{|c|}{ Agisoft } \\
\hline \multicolumn{1}{|c|}{ Parameter } & Mean value & Standard deviation \\
\hline $\mathbf{f}[\mathbf{m m}]$ & 14.857 & 0.088 \\
$\mathbf{x}_{\mathbf{0}}$ [mm] & 11.444 & 0.133 \\
$\mathbf{y}_{\mathbf{0}}$ [mm] & 7.521 & 0.103 \\
$\mathbf{K}_{\mathbf{1}}$ & $-3.02 \mathrm{E}-04$ & $3.45 \mathrm{E}-04$ \\
$\mathbf{K}_{\mathbf{2}}$ & $2.31 \mathrm{E}-03$ & $7.12 \mathrm{E}-04$ \\
$\mathbf{K}_{\mathbf{3}}$ & $-5.62 \mathrm{E}-03$ & $5.21 \mathrm{E}-03$ \\
$\mathbf{P}_{\mathbf{1}}$ & $-8.00 \mathrm{E}-07$ & $1.78 \mathrm{E}-07$ \\
$\mathbf{P}_{\mathbf{2}}$ & $2.79 \mathrm{E}-06$ & $9.7 \mathrm{E}-07$ \\
\hline
\end{tabular}

Table 3. The results of Sony NEX 5R calibration (Matlab and Agisoft)

\begin{tabular}{|l|c|c|}
\hline \multicolumn{3}{|c|}{ Matlab } \\
\hline \multicolumn{1}{|c|}{ Parametr } & Mean value & Standard deviation \\
\hline $\mathbf{f}[\mathbf{m m}]$ & 14.487 & 0.083 \\
$\mathbf{x}_{\mathbf{0}}[\mathbf{m m}]$ & 11.467 & 0.120 \\
$\mathbf{y}_{\mathbf{0}}[\mathbf{m m}]$ & 7.351 & 0.125 \\
$\mathbf{K}_{\mathbf{1}}$ & $-3.44 \mathrm{E}-04$ & $1.43 \mathrm{E}-04$ \\
$\mathbf{K}_{\mathbf{2}}$ & $-5.38 \mathrm{E}-06$ & $1.47 \mathrm{E}-03$ \\
$\mathbf{K}_{\mathbf{3}}$ & $1.28 \mathrm{E}-03$ & $4.61 \mathrm{E}-03$ \\
$\mathbf{P}_{\mathbf{1}}$ & $-1.62 \mathrm{E}-05$ & $1.17 \mathrm{E}-05$ \\
$\mathbf{P}_{\mathbf{2}}$ & $2.30 \mathrm{E}-05$ & $1.40 \mathrm{E}-05$ \\
\hline \multicolumn{2}{|c|}{ Agisoft } & \\
\hline $\mathbf{P a r a m e t r}_{\mathbf{f}}$ & Mean value & Standard deviation \\
\hline $\mathbf{f}[\mathbf{m m}]$ & 14.589 & 0.153 \\
$\mathbf{x}_{\mathbf{0}}[\mathbf{m m}]$ & 11.363 & 0.102 \\
$\mathbf{y}_{\mathbf{0}}[\mathbf{m m}]$ & 7.411 & 0.093 \\
$\mathbf{K}_{\mathbf{1}}$ & $-3.29 \mathrm{E}-04$ & $5.34 \mathrm{E}-04$ \\
$\mathbf{K}_{\mathbf{2}}$ & $3.68 \mathrm{E}-03$ & $9.44 \mathrm{E}-03$ \\
$\mathbf{K}_{\mathbf{3}}$ & $-2.53 \mathrm{E}-02$ & $6.10 \mathrm{E}-02$ \\
$\mathbf{P}_{\mathbf{1}}$ & $-1.19 \mathrm{E}-05$ & $1.04 \mathrm{E}-05$ \\
$\mathbf{P}_{\mathbf{2}}$ & $4.89 \mathrm{E}-06$ & $1.11 \mathrm{E}-05$ \\
\hline
\end{tabular}

Table 4. The results of Sony NEX 5T calibration (Matlab and Agisoft) 
Focal length values for Sony Nex 5R camera (theoretical and calculated) varies by $0.1 \mathrm{~mm}$. It is a small difference, most likely due to application errors.

However, in the case of calibration parameters for camera Sony NEX 5T, the results are very different from the other, eg . focal length is different from that specified by the manufacturer to 0.5 $\mathrm{mm}$ for Matlab and $0.4 \mathrm{~mm}$ for Agisoft.

\section{CONSLUSIONS}

The paper presents the process, the results and the accuracy of two calibrations methods. After the software processing, the camera calibration parameter values were obtained. the analyzes show that the most accurate deployed software program was Image Master . It achieved the highest accuracy of all programs for the majority of calibration parameters .

for the calibration of digital cameras have been used for nonmetric photogrammetry typically best results (stability and accuracy has been obtained I Image Master Calib. Also processing time and complexity of calculations is the best using this sotfware.

\section{ACKNOWLEDGEMENTS}

This work was supported by grand co-financed with Faculty of Civil Engineering and Geodesy Military university of Technology (Geodesy Institute).

\section{REFERENCES}

Dabrowski, R., Delis, P., Wyszynski, M., 2014. Mare Analysis of the possibility of using a video camera as a UAV sensor, Conference: 9th International Conference on Environmental Engineering (ICEE) Location: Vilnius, LITHUANIA

Delis P., Fryskowska A., Kedzierski M., Wilinska M., 2013. 3D modeling of architectural objects from video data obtained with the fixed focal length lens geometry. Geodesy and Cartography Vol. 62, No 2, pp. 123-138 DOI: 10.2478/geocart-2013-0007

Douterloigne, Koen, Sidharta Gautama, and Wilfried Philips. 2009. Fully automatic and robust UAV camera calibration using chessboard patterns. Geoscience and Remote Sensing Symposium, 2009 IEEE International, IGARSS 2009. Vol. 2. IEEE.

Fraser, C., Remondino, F., 2006. Digital camera calibration methods: considerations and comparisons. IAPRS Volume $X X X V I$, Part 5, Dresden 25-27.

Harley I., Kyle S., Luhmann T., Robson S., 2009. Close Range Photogrammetry Principles, Methods and Applications, pp. 97148, 449-459.

Hastedt, H., and T. Luhmann. 2015. "Investigations on the quality of the interior orientation and its impact in object space for UAV photogrammetry." The International Archives of Photogrammetry, Remote Sensing and Spatial Information Sciences 40.1 (2015): 321.

Honkavaara, E., Ahokas, E. Hyyppä, J., Jaakkola, J., Kaartinen, H., Kuittinen, R., Markelin, L. and Nurminen, K. 2006. Geometric test field calibration of digital photogrammetric sensors. ISPRS Journal of Photogrammetry \& Remote Sensing.60, pp. 387-399.

Karras, G.E. and Mavrommati, D. 2001. Simple calibration techniques for non-metric cameras. CIPA International Symposium, Potsdam, Germany, pp. 18-21.

Kedzierski, M., Wierzbicki, D., 2015. Radiometric quality assessment of images acquired by UAV's in various lighting and weather conditions in Measurement Volume: 76, Pages: 156169.

Kedzierski, M., Fryskowska, A., 2008. Precise method of fisheye lens calibration. Proceedings of the ISPRS-Congress, Beijing, China.

Nikon, 2016. Geospatial Modeling \& Visualization http://gmv.cast.uark.edu/photogrammetry/photogrammetryhardware/nikon-d200-ir/

Pérez, M., Agüera F., and Carvajal. F., 2011. Digital camera calibration using images taken from an unmanned aerial vehicle." Conference on Unmanned Aerial Vehicle in Geomatics, Zurich, Switzerland. Vol. 20.

Rodríguez, B., Díaz, D., Haydeé, S., García, G., and Benítez Piñero Y., 2008. Experience using non-metric cameras in photogrammetry. International Archives of the Photogrammetry, Remote Sensing and Spatial Information Sciences 37, pp. 769-772.

Wang, A., Qiu ,T., Shao, L., 2009. A Simple Method of Radial Distortion Correction with Centre of Distortion Estimation, Journal of Mathematical Imaging and Vision 35: 165-172 DOI 10.1007/s10851-009-0162-1.

Revised June 2015 